\title{
Acidente vascular encefálico associado à síndrome do roubo da subclávia: relato de caso clínico
}

\author{
Stroke associated with subclavian robbery syndrome: clinical case report
}

Accidente cerebrovascular asociado al síndrome de robo subclavio: reporte de caso clínico

Aimée Murad Pinton ${ }^{1 *}$, Thaisa Mourão Vasconcelos de Mattos.

\section{RESUMO}

Objetivo: Relatar um caso singular de um paciente com Acidente Vascular Encefálico (AVE) associado à síndrome do roubo da subclávia, aumentando assim a suspeição clínica diagnóstica precoce, limitar complicações e diminuir agudizações. Detalhamento do caso: Paciente masculino, 58 anos, antecedente de labirintopatia, deu entrada em um hospital do interior paulista após quadro de cefaleia, rouquidão, vertigem rotacional, cianose em membro superior esquerdo e alteração de pressão arterial média em membros superiores, sendo aventada a hipótese de Acidente Vascular Cerebral (AVC) associado à Síndrome do Roubo da Subclávia. Exames de imagem mostraram áreas injúrias agudas e subagudas com componente hemorrágico e perda de flow-void em segmentos da artéria vertebral esquerda. O paciente foi submetido a tratamento clínico-cirúrgico, apresentando retorno dos pulsos de membro superior, manteve cianose em quinto dedo de mão esquerda e ataxia de membro superior esquerdo, vertigem ocasional e diminuição progressiva de rouquidão, sendo encaminhado para seguimento. Considerações finais: Por ser uma doença relativamente rara, reconhecer sinais e sintomas de insuficiência vertebrobasilar e uma história clínica detalhada, se faz necessária para um diagnóstico precoce, buscando limitar complicações e diminuir episódios de agudização ao longo dos anos, além de favorecer melhor estratégia de tratamento a cada paciente.

Palavras-chave: Acidente vascular encefálico, Síndrome do roubo subclávio, Insuficiência vertebrobasilar, Vertigem.

\begin{abstract}
Objective: To report a unique case of a patient with cerebrovascular accident (CVA) associated with subclavian steal syndrome, thus increasing early diagnostic clinical suspicion, limiting complications and decreasing exacerbations. Case details: A 58-year-old male patient with a history of labyrinth disease was admitted to a hospital in the interior of São Paulo after to have headache, hoarseness, rotational vertigo, cyanosis in the left upper limb and change in mean arterial pressure in the upper limbs, being considered hypothesis of stroke associated with subclavian steal syndrome. Imaging exams showed areas of acute and subacute injuries with a hemorrhagic component and loss of flow-void in segments of the left vertebral artery. The patient underwent clinical-surgical treatment, with return of pulses in the upper limb, remained cyanosis in the fifth finger of the left hand and ataxia of the left upper limb, occasional vertigo and progressive decrease in hoarseness, being referred for follow-up. Final considerations: Because it is a relatively rare disease, recognizing signs and symptoms of vertebrobasilar insufficiency and a detailed clinical history is necessary for an early diagnosis, seeking to limit complications and reduce episodes of exacerbation over the years, in addition to favoring a better strategy of treatment for each patient.
\end{abstract}

Keywords: Stroke, Subclavian steal syndrome, Vertebrobasilar insufficiency, Vertigo.

${ }^{1}$ Santa Casa de Misericórdia de Franca, Franca - SP. *E-mail: aimeemuradpinton@gmail.com 


\section{RESUMEN}

Objetivo: Reportar un caso único de un paciente con accidente cerebrovascular (ACV) asociado a síndrome de robo de subclavia, aumentando así la sospecha clínica diagnóstica precoz, limitando las complicaciones y disminuyendo las exacerbaciones. Detalles del caso: Un paciente masculino de 58 años con antecedentes de enfermedad del laberinto ingresó en un hospital del interior de São Paulo después de un cuadro de dolor de cabeza, ronquera, vértigo rotacional, cianosis en el miembro superior izquierdo y alteración de la presión arterial media en el miembros superiores, considerándose la hipótesis de Accidente Cerebrovascular (ACV) asociado a síndrome de robo de subclavia. Los exámenes de imagen mostraron áreas de lesiones agudas y subagudas con componente hemorrágico y pérdida de vacío de flujo en segmentos de la arteria vertebral izquierda. El paciente fue sometido a tratamiento clínico-quirúrgico, con retorno de pulsos en miembro superior, permaneciendo cianosis en quinto dedo de mano izquierda y ataxia de miembro superior izquierdo, vértigo ocasional y disminución progresiva de ronquera, siendo remitido para seguimiento. Consideraciones finales: Por ser una enfermedad relativamente rara, es necesario el reconocimiento de signos y síntomas de insuficiencia vertebrobasilar y una historia clínica detallada para un diagnóstico precoz, buscando limitar las complicaciones y disminuir los episodios de agudización a lo largo de los años, además de favorecer una mejor estrategia de tratamiento tratamiento para cada paciente.

Palabras clave: Accidente cerebrovascular, Síndrome del robo de la subclavia, Insuficiencia vertebrobasilar, Vértigo.

\section{INTRODUÇÃO}

A Síndrome do Roubo da Subclávia (SRS) é causada por uma alteração vascular, onde ocorre inversão do fluxo de sangue da artéria vertebral decorrente de oclusão ou estenose da artéria subclávia, do mesmo lado, isso gera um enchimento retrógrado da artéria subclávia e pode ocasionar sintomas decorrentes da hipoperfusão cerebral (POLLARD H, et al., 1998; PASSOS MD, et al., 2016; LOPES MNSC, et al., 2013; TAN X, et al., 2017).

É uma afecção relativamente rara de etiologia múltipla e associada ao tabagismo em $78 \%$ a $100 \%$ dos casos, como também a doença arterial coronariana em $27 \%$ a $65 \%$ dos casos. Apresenta uma discreta prevalência no sexo masculino, próximo da sexta década de vida, sendo observada em até $6 \%$ dos pacientes com sopro cervical na forma assintomática. Dentre as causas a arterioesclerose é a principal causa de doença oclusiva associada à síndrome, outras causas são inflamatória, congênita, embólica, pós-operatórios e traumática (PASSOS MD, et al., 2016; POTTER BJ e PINTO DS, 2014).

Essa obstrução ocorre principalmente na região proximal da artéria subclávia esquerda. Zimmerman NB (1993) observou uma proporção de 3:1 de lesões sintomáticas da artéria subclávia do lado esquerdo em comparação à direita. A obstrução gera uma menor pressão na artéria subclávia ipsilateral, o que faz o sangue percorrer de forma retrógrada a artéria vertebral contralateral, passar pela artéria basilar até alcançar a artéria vertebral ipsilateral à obstrução, gerando uma circulação colateral. Esse fluxo contrário ocorre em cerca de $90 \%$, de forma contínuo ou ocasional, nos pacientes com uma obstrução moderada maior que $50 \%$. Com o fluxo sanguíneo retrógrado o sangue do sistema basilar é sequestrado, o que pode diminuir e comprometer a irrigação encefálica, já que cerca de $20 \%$ do suprimento sanguíneo cerebral vem das artérias cerebrais (PASSOS MD, et al., 2016; POTTER BJ e PINTO DS, 2014).

É uma doença em sua maioria assintomática, principalmente quando o suprimento sanguíneo colateral consegue suprir as necessidades, por sua vez quando essa compensação deixa de existir os sintomas, devido à hipoperfusão cerebral vertebrobasilar e isquemia de membro superior, começam a aparecer (FREGNIL F, et al., 2003). Heidrich H (1969) avaliou as manifestações de acordo com a localidade como cerebral, cerebral e queixas de membros superiores, apenas membros superiores e assintomáticos, sendo as principais manifestações cerebrais tontura $75 \%$, cefaleia $43 \%$, distúrbio visual $35 \%$, síncope $25 \%$, ataxia $11 \%$, distúrbios 
de fala $11 \%$ e queixas relacionada ao membro $15 \%$. Smith JM (1994), por sua vez, seguiu por 14 anos um grupo de pacientes com sintomas secundários da síndrome, sendo os mais comuns com o tempo vertigem, síncope e fraqueza (MOREIRA DA, et al. 2006).

A primeira visualização angiográfica ocorreu em 1960, porém a aplicação da síndrome, no meio científico, ocorreu somente em 1961, após o relato de sinais de insuficiência vascular cerebral em associação a inversão do fluxo da artéria vertebral, como consequência da obstrução da artéria subclávia. Já o termo "síndrome do roubo da subclávia" foi introduzido em 1962 em um editorial do New England Journal of Medicine. Apesar da ampla utilização do termo Síndrome do Roubo da Subclávia somente deve ser aplicado, quando a inversão no fluxo na artéria vertebral ocasionar sintomas de hipoperfusão vertebro-basilar e/ou de isquemia do membro superior, em quadros assintomáticos, intermitentes ou ocultos, o mais correto seria usar somente a expressão roubo da subclávia, não utilizando o termo síndrome (BERNADES CHA, et al., 1999; PASSOS MD, et al., 2016).

A suspeita diagnóstica é feita através da história clínica e exame físico compatível, onde pode ocorrer relatos como vertigem, síncope, claudicação intermitente de membro superior e no exame físico pode ser observado, entre outros, diferenças de pressão arterial sistólica e/ou pulsos entre os membros, presença de sopros carotídeos e supra escapulares (MOREIRA DA, et al., 2006). Já em relação aos exames complementares em busca do diagnóstico, a ultrassonografia com Doppler é um método não invasivo e sensível, outro método a ser considerado é a angiografia (LOPES MNSC, et al., 2013). O tratamento invasivo quando instituído é realizado, tradicionalmente, com a abordagem cirúrgica, sendo outras alternativas a angioplastia transluminal percutânea e o implante de stents, o objetivo é restaurar o fluxo normal da artéria vertebral, acabar com a hipoperfusão cerebral e a clínica ocasionada por essa (PASSOS MD, et al., 2016).

O objetivo deste estudo foi relatar um caso relativamente raro e singular de um paciente com acidente vascular encefálico (AVE) associado à síndrome do roubo da subclávia, aumentando assim a suspeição clínica diagnóstica precoce, limitar complicações, diminuir agudizações e favorecer estratégias de tratamento, bem como contribuir com o número de casos relatados e futuras pesquisas, devido ao reduzido número de artigos recentes encontrados.

\section{DETALHAMENTO DO CASO}

Os dados coletados, informações descritas, exames de imagens e relato clínico foram autorizados pelo paciente com assinatura do Termo de Consentimento Livre Esclarecido (TCLE), descrito conforme orientações do Comitê de Ética em Pesquisa (CEP) e aprovado sob o número de Certificado de Apresentação de Apreciação Ética (CAAE) 52599221.9.0000.5438.

Paciente do sexo masculino, 58 anos, branco, antecedente de labirintopatia em uso de dicloritrato de betaistina, uso irregular em crises, não etilista, não tabagista, deu entrada em um hospital do interior paulista, após quadro de cefaleia súbita a esquerda, rouquidão, cervicalgia, vertigem rotacional, ataxia e cianose em membro superior esquerdo, principalmente em região de 4 e 5 dedos sem melhora com repouso ou medicações.

Em exame físico observou-se paciente consciente, orientado, rouquidão, paralisia facial à esquerda, ataxia de membro superior esquerdo, cianose de dedos da mão esquerda, em especial 4 e 5 dedos, diminuição do pulso braquial e radial esquerdo e alteração de pressão arterial média sendo 150/80 mmhg a direita e 120/70 mmhg membro superior esquerdo, sem sopros cardíacos ou sopros cervicais audíveis.

Exame de imagem inicial a ressonância magnética mostrou, entre outros achados, áreas de restrição a difusão das moléculas de água, com componente hemorrágico associado, envolvendo porção inferior do hemisfério cerebelar à esquerda, bem como porção póstero-lateral da região bulbar, compatíveis com injúrias isquêmicas agudas/subaguda e perda de flow-void habitual do segmento V4 e parte seguimento V3 da artéria vertebral esquerda. 
Figura 1 - Restrição a difusão das moléculas de água, com componente hemorrágico em hemisfério cerebelar à esquerda, região bulbar e perda de flow-void habitual de artéria vertebral esquerda.

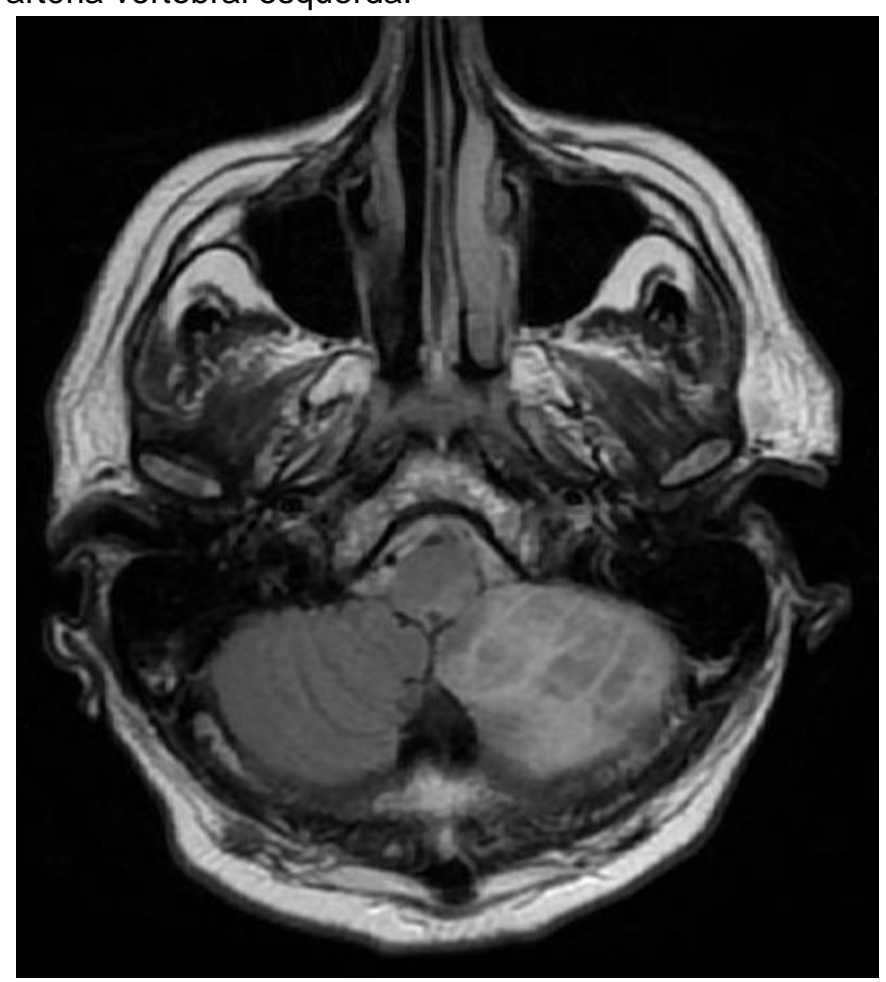

Fonte: Pinton AM e Mattos TMV, 2022.

Realizado posteriormente a arteriografia, que evidenciou a presença de trombose no segmento proximal de artéria subclávia esquerda, com fluxo preservado e trombose na origem e segmento proximal de artéria vertebral esquerda, sem fluxo em território cerebral, sendo aventada então a hipótese de Acidente vascular encefálico com transformação hemorrágica associado a Síndrome do Roubo da Subclávia.

Figura 2 - Trombose no segmento proximal de artéria subclávia esquerda e segmento proximal de artéria vertebral esquerda, ausência de fluxo em território cerebral.

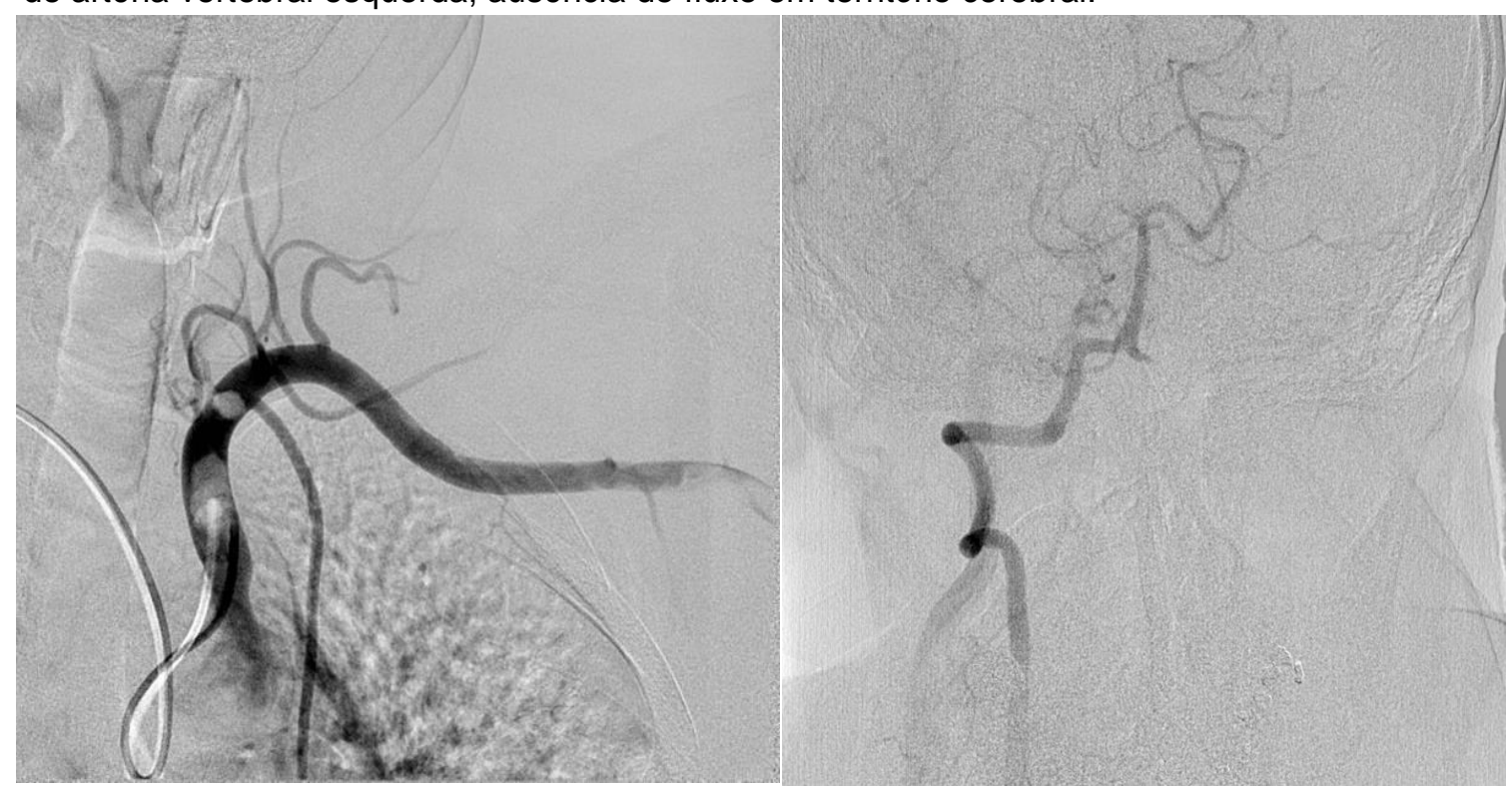

Fonte: Pinton AM e Mattos TMV, 2022. 
Devido ao quadro clínico e sintomatologia associada foi iniciado estatina $40 \mathrm{mg} / \mathrm{dia}$ e dimenidrinato 100 mg 6/6h e quatro dias após admissão hospitalar, em condutas associadas a equipe de cirurgia vascular, foi realizado procedimento cirúrgico de derivação axilo-axilar com safena invertida. No decorrer da internação paciente evoluiu com melhora dos pulsos braquial, radial e ulnar a esquerda mantendo cianose em topografia distal de 5 dedo à esquerda, vertigem ocasional, ataxia de membro superior esquerdo e diminuição progressiva de rouquidão, ainda durante o período de internação foi realizado o diagnóstico de diabetes mellitus tipo 2.

O paciente em questão recebeu alta hospitalar após estabilização clínica no oitavo dia de internação, sendo optado, juntamente com a equipe de cirurgia vascular, devido quadro clínico e o AVC com transformação hemorrágica por medicações orais como ácido acetilsalicílico $100 \mathrm{mg} / \mathrm{dia}$, clopidogrel 75 $\mathrm{mg} / \mathrm{dia}$, sinvastatina $40 \mathrm{mg} / \mathrm{dia}$ e metformina $500 \mathrm{mg} \mathrm{2x/dia} \mathrm{além} \mathrm{de} \mathrm{encaminhamento} \mathrm{com} \mathrm{equipe} \mathrm{de}$ neurologia, cirurgia vascular e fisioterapia.

\section{DISCUSSÃO}

A síndrome do roubo da subclávia é relativamente rara, sendo a maioria dos pacientes assintomáticos, em especial quando o suprimento sanguíneo é compensado pela artéria vertebral contralateral ou pelo sistema carotídeo no polígono de willis 4 (DUBIN IMD, et al., 2020; FREGNIL F, et al., 2003).

Entre os fatores de riscos associados à síndrome o tabagismo, presente em 78 a $100 \%$ dos casos, e a doença arterial coronariana em 27 a 65\% são bem estabelecidos (PASSOS MD, et al., 2016). Outros estudos compararam os fatores de risco para doença cardiovascular com a ocorrência de estenose subclávia, sendo comprovado a associação entre hipertensão arterial, diabetes mellitus, história de tabagismo maior que 10 anos-maço, níveis elevados de interleucina-6, fator VIIlc e proteína C-reativo (LAK HM, et al., 2020; SHADMAN R, 2004). O paciente em questão apresentava entre os fatores de risco fator a diabetes mellitus, essa com o diagnóstico intra-hospitalar.

Os sintomas podem aparecer principalmente quando atividades com membro superior desviam ou acentuam o desvio de fluxo sanguíneo cerebral, acentuando a isquemia, entre os sintomas se destacam tontura, cefaleia, distúrbios visuais, ataxias como também claudicação e fadiga do membro (FREGNIL F, et al., 2003; LAK HM, et al., 2020).

A suspeita diagnóstica se baseia principalmente na sintomatologia e no exame físico, onde observamos sintomas relacionados à insuficiência vértebro-basilar e isquemia de membro superior. Cerca de um terço dos pacientes apresentaram algia, parestesia ou claudicação no braço, desses apenas $5 \%$ apresentaram sintomas isquêmicos graves ou embolia em ramos arteriais distais dos membros superiores, estando a diminuição de pulsos radiais presentes em $85 \%$ no braço envolvido (FREGNIL F, et al., 2003). Sopros podem ou não ser observados, estando relacionados ao grau de obstrução, já a diferença de pressão sistólica maior que $20 \mathrm{mmhg}$ apresenta sensibilidade e especificidade significativa sendo 84 e 95\% respectivamente para obstrução (FREGNIL F, et al., 2003; DUBIN IMD, et al., 2020).

A clínica e o exame físico do paciente aventava a diagnóstica de acidente vascular encefálico relacionado a síndrome do roubo da subclávia, pois entre esses se destacavam vertigem, cefaleia, rouquidão, ataxia de membros superior esquerdo, cianose de dedos da mão com diminuição do pulso braquial e radial a esquerda e alteração de pressão arterial sistólica $>20 \mathrm{mmhg}$ entre os membros, não sendo observados sopros cardíacos ou cervicais.

Hennerici M, et al. (1988) concluíram em seus estudos que o roubo da subclávia raramente estava associada a déficits neurológicos, de acordo com esses, seria na verdade um marcador da existência de doença aterosclerótica grave craniana, coronária e vascular periférica e os eventos neurológicos que poderiam vir a ocorrer seriam relacionados a essa doença aterosclerótica difusa (FREGNIL F, et al., 2003).

Estudos mais recentemente realizados colocam como principais fatores associados ao risco de Acidente Vascular Cerebral (AVC) a presença de sintomas neurológicos, esses equivalentes a $86 \%$ do risco, outros são estenose vertebral grave em $58 \%$ e estenose arterial intracraniana grave em $24 \%$, não foi observado 
associação entre idade, alteração de pressão arterial sistólica $>20 \mathrm{mmhg}$ entre os membros, tratamento invasivo ou piora dos sintomas durante acompanhamento com o aumento de riscos de AVC. Entre os riscos cardiovasculares associados ao risco de AVC na síndrome do roubo da subclávia, apenas a hipertensão arterial sistêmica se mostrou associada (TAN X et al., 2017). Uma provável causa para esses quadros é a progressão da doença aterosclerótica, trombose ou ateroembolização (LOPES MNSC, et al., 2013). A arteriografia realizada nos mostrou trombose de artéria vertebral esquerda, como também em segmento proximal de artéria subclávia esquerda.

A triagem diagnóstica em casos suspeitos pode ser realizada com dopplerfluxometria e/ou eco-color doppler, exames esses com alta precisão e não invasivos, mas a arteriografia digital permanece como exame solicitado para diagnóstico definitivo, principalmente em casos duvidosos, é necessário porém se atentar pois já foi demonstrado que após uso de contraste o fluxo da artéria vertebral pode inverter, apesar da ausência de lesão estenosante em subclávia, o que não constitui a síndrome do roubo da subclávia (LOPES MNSC, et al., 2013).

O tratamento é abordado de várias formas sendo pautado pela sintomatologia, comorbidades e suporte hospitalar dos casos de pacientes que apresentam a Síndrome do Roubo da Subclávia, sendo relatadas desde condutas conservadoras, com controle e tratamento de fatores de risco e comorbidades, a invasivas, essa questionável em pacientes assintomáticos (LAK HM, et al., 2020). Entre exemplos de condutas invasivas pode ser realizado Bypass carotídeo-subclávio, Transposição carótida subclávia, bypass Axilo-axilar, Endarterectomia carotídea, Aterectomia e Angioplastia transluminal percutânea (PTA) com implante de stent o objetivo é sempre restaurar o fluxo, sanando a hipoperfusão cerebral e de membro (FREGNIL F, et al., 2003; PASSOS MD, et al., 2016).

As complicações que podem ser observadas em procedimentos cirúrgicos invasivos estão muitas vezes associadas a doenças coronarianas e cerebrovasculares coexistentes, já complicações relacionadas ao ato cirúrgico são infecções, trombose, reestenoses entre outros. A PTA quando associada ao implante de stent parece ter melhores resultados do que usada de forma isolada (FREGNIL F, et al., 2003; PASSOS MD, et al., 2016).

Pelo exposto como a maioria dos casos de Síndrome do Roubo da Subclávia é assintomática e sendo uma doença relativamente rara, reconhecer sinais e sintomas de insuficiência vertebrobasilar e de membros superiores, quando presentes, além de uma história clínica detalhada se faz necessária no escopo buscando um diagnóstico precoce de doença, limitar complicações e diminuir episódios de agudização ao longo dos anos, assim como fornecer o acesso a melhor estratégia de tratamento individualizada e indicada a cada paciente, de acordo com o momento da doença, condições clínicas e complicações destas apresentadas.

\section{REFERÊNCIAS}

1. BERNADES CHA, et al. Síndrome do roubo da subclávia: relato de caso. Acta Medica Misericordiae, 1999; 2(I): 3841.

2. DUBIN IMD, et al. Subclavian Steal Syndrome, Cerebellar Infarctions, and Alcohol. The American Journal of Medicine, 2020; 134(5): 317-318.

3. FREGNIL F, et al., Tratamento de síndrome de roubo de subclávia com angioplastia transluminal percutânea e stenting: Relato de caso. Arq. Neuro-Psiquiatr., 2003; 61(1).

4. HEIDRICH H, BAYER O. Symptomatology of the Subclavian Steal Syndrome. Sage journals, 1969; 20(7):406-413.

5. HENNERICI M, et al. The subclavian steal phenomenon: a common vascular disorder with rare neurologic deficits. Revista eletrónica Neurology, 1988; 38(5): 669-673.

6. LACEY KO. Subclavian steal syndrome: a review. J. Vasc. Nurs.,1996; 15(1): 1-7

7. LAK HM, et al. Coronary Subclavian Steal Syndrome: A Contemporary Review. Revista eletrónica Cardiology, 2020.

8. LOPES MNSC, et al. Síndrome do roubo subclávio: relato de caso. Revista eletrônica Brasília Med., 2013; 50(2): 174177.

9. MOREIRA DA, et al. Reabilitação Vestibular em Síndrome do Roubo da Subclávia Vestibular, Relato de caso. Arq. Int. Otorrinolaringol., 2006; 10(2): 148-153. 
10. PASSOS MD, et al. Atualização sobre Ultrassom Doppler das Artérias Vertebrais: Síndrome do Roubo da Subclávia. Arq. Bras. Cardiol.: Imagem cardiovasc., 2016; 29(2): 58-62.

11. POLLARD H, et al. Subclavian steal syndrome: a review. Australas Chiropr Osteopathy, 1998; 7(1): 20-28.

12. POTTER BJ, PINTO DS. Subclavian Steal Syndrome. Revista eletrônica Circulation, 2014; 129: 2320-2323.

13. SHADMAN R, et al. Subclavian artery stenosis: prevalence, risk factors, and association with cardiovascular diseases. Revista eletrônica J. Am. Coll. Cardiol., 2004; 44(3): 618-623.

14. SMITH JM, et al. Subclavian steal syndrome. A review of 59 consecutive cases. J. Cardiovasc. Surg., 1994; 35(1): 1114.

15. TAN X, et al. Risk of stroke in imaging-proven subclavian steal syndrome. Journal of Clinical Neuroscience, 2017.

16.ZIMMERMAN NB. Occlusive vascular disorder of the upper extremity. Hand Clin., 1993; 9(1): 139-150. 